\title{
Highly expressed ribosomal protein L34 predicts poor prognosis in acute myeloid leukemia and could be a po- tential therapy target
}

\author{
Liang Wang ${ }^{\mathrm{a},},{ }^{,}$, Jing Yang ${ }^{\mathrm{a}}$, Henan Wang ${ }^{\mathrm{a}}$, Weida Wang ${ }^{\mathrm{b}}$, Xiaojie Liang ${ }^{\mathrm{c}}$ \\ ${ }^{a}$ Department of Hematology, Beijing Tongren Hospital, Capital Medical University, Beijing, 100730, China. \\ ${ }^{b}$ Department of Hematologic Oncology, Sun Yat-sen University Cancer Center, Guangzhou, 510000, China. \\ 'Second Clinical Medical College of Southern Medical University, Guangzhou, 510280, China.
}

\begin{abstract}
Background: Acute myeloid leukemia (AML) is a devastating malignancy with great heterogeneity, novel prognostic biomarkers and therapy targets are needed to improve the precise management of AML patients. Increasing evidence has shown the role of RPL34, a ribosomal protein, in tumorigenesis and progression. However, the detailed expression status and clinical significance of RPL34 in AML are largely unknown.

Methods: The expression level of RPL34 was detected in bone marrow samples from both AML patients and AML cell lines. Then using recombinant shRNA-lentiviral vector, we analyzed the impact of RPL34 knockdown on cell proliferation, apoptosis and cell cycle distribution. Lastly, by analyzing public gene expression datasets (GSE12417 and GSE2191), we determined the prognostic role of RPL34 in AML.

Results: The mRNA level of RPL34 was significantly elevated in AML bone marrow samples and cell lines. Patients with high level of RPL34 had inferior survival outcomes than their counterparts, and upregulation of RPL34 may mediate chemoresistance in AML. Through knockdown of RPL34 in HL-60 cell line, we found cell proliferation was inhibited, cell apoptosis was triggered, and cell cycle was arrested in S phase.

Conclusions: The present study demonstrated that downregulation of RPL34 could inhibit cell proliferation, promote cell apoptosis, and induce cell cycle arrest in AML cell line HL-60. Also, its expression and clinical significance in AML patients was confirmed. All these findings suggest that RPL34 may be a potential novel therapy target in AML.
\end{abstract}

Keywords: Acute myeloid leukemia, ribosomal protein L34, biomarker, therapy target, chemoresistance

\section{Introduction}

Acute myeloid leukemia (AML) is the most common type of acute leukemia among adults, with the median age of diagnosis being 67 years [1]. A combination of cytarabine and an anthracycline (daunorubicin or idarubicin, the so called $7+3$ regimen) has been the standard induction regimen for more than four decades. This $7+3$ regimen can result in a complete remission (CR) rate of $60-85 \%$ in younger patients and only $40-60 \%$ in patients aged 60

\footnotetext{
* Corresponding author: Liang Wang

Mailing address: Department of Hematology, Beijing Tongren Hospital, Capital Medical University, Beijing, 100730, China. Email: wangliangtrhos@126.com
}

Received: 20 March 2020 / Accepted: 23 March 2020 years or older [2]. Moreover, several older patients, especially those with poor performance status, cannot tolerate the toxicities caused by this $7+3$ regimen. Although standard induction chemotherapy can induce high remission in most AML patients, more than $30 \%$ of patients are refractory to treatment, and nearly half of patients eventually relapsed and died of AML [3]. Thus, novel agents are urgently needed to improve the outcome of patients with AML. In recent years, some novel agents with different mechanisms of action have been demonstrated to be active in specific cohort of patients with AML, such as FLT3 inhibitors [4], IDH2 inhibitors [5], and BCL2 inhibitors [6]. Previously, our group used a cDNA microarray to explore the differently expressed genes between AML samples and normal bone marrow (BM) samples and found RPL34 highly expressed in AML patients. Ribosomal proteins (RPs), as components of ribosomes, function in the process of protein translation and ribosome assembly, which are critical for the proliferation and survival of all cells. In 
recent years, deregulation of RPs has been found to be related with various disease including tumor. For example, in gastric cancer cells, upregulated RPL6 and RPS13 can accelerate cell proliferation and promote cell cycle progression, while increased expression of RPS3 and RPL23 can induce drug resistance [7]. All these findings indicate that RPs may be used as valuable biomarkers and predictors for cancer diagnosis and treatment efficacy. Thus, investigation of the role of RPs may promote the development of novel targeted therapies for various cancers. As a ribosomal protein, RPL34 has also been reported to play important roles in tumorigenesis [8]. However, the biological function and clinical significance of RPL34 in AML remains largely unknown. In the present study, we firstly demonstrated high expression of RPL34 in AML cell lines, and then using lentivirus-delivered small interfering RNA (siRNA) technique, we examined the effect of RPL34 knockdown on AML cell growth in vitro. Moreover, we explored the prognostic role and gene interactions of RPL34 by analyzing a public gene expression database of AML.

\section{Materials and methods}

\section{Patients and collection of BM samples}

Ten patients diagnosed as de novo AML were included in this study. Eleven patients diagnosed as non-AML disease with normal bone marrow (BM) examination results were used as controls (BM examination was done in these patients due to suspicion of hematologic disease, but finally ruled out the diagnosis of hematologic disease). BM samples were obtained from all these patients before treatment. The collection and use of BM samples was approved by the ethic committee of Sun Yat-sen University Cancer Center and all patients provided written informed consent.

\section{Cell lines}

Human acute myeloid leukemia cell lines HL-60 and KG-1 were purchased from Shanghai Cell Bank (Shanghai, China), and cultured in RPMI-1640 medium, supplemented with $20 \%$ fetal bovine serum (FBS), 100U/ml penicillin and $100 \mu \mathrm{g} / \mathrm{ml}$ streptomycin at $37^{\circ} \mathrm{C}$ in a $5 \% \mathrm{CO}_{2}$ incubator.

\section{Quantitative RT-PCR}

Total RNA from BM mononuclear cells and 2 cell lines, HL-60 and KG-1, was extracted using TRIzol reagent (Invitrogen, Shanghai, China). Then $2 \mu \mathrm{g}$ of total RNA from each sample was reverse transcribed to single-stranded cDNA, $1 \mu \mathrm{L}$ of which was used as the template for PCR experiment. The primers used in this study were as follows: RPL34 forward, 5'-GTT TGA CAT ACC GAC GTA GGC-3' and reverse, 5'-GCA CAC ATG GAA CCA CCA TAG-3'; GAPDH forward, 5'-TGA CTT CAA CAG CGA CAC CCA-3' and reverse, 5'-CAC CCT GTT GCT GTA GCC AAA-3'. All samples were detected in triplicate.

\section{Recombinant lentiviral vectors and cell transfection}

We designed a siRNA (CACAGAGTCAGAAAGCTAA) to target the RPL34 transcript (GenBank accession No. NM_000995) and a scrambling sequence (TTCTCCGÄACGTGTCACGT) as negative control (NC). Then a short hairpin RNA (shRNA) was synthesized by GeneChem Co.Ltd (Shanghai, China) based on the above siRNA sequence (designated as shRPL34 and shCtrl), and inserted into a linearized vector GV115 with green fluorescent protein (GFP) gene and AgeI/EcoRI sites to establish the shRNA lentiviral vectors. Using Lipofectamine 2000 (Invitrogen, Carlsbad, US), we co-transfected these two shRNA lentiviral vectors into a tool cell line, 293T cells, with lentiviral helper plasmids pHelper 1.0 and pHelper 2.0 (GeneChem Co.Ltd, Shanghai, China). Then the HL-60 cells were separately transfected with shRPL34 or shCtrl lentivirus.

\section{Cell proliferation assay}

10 days after transfection with shRPL34 or shCtrl lentivirus, the HL-60 cells were seeded at a density of 2000 cells/well in 96-well plates and incubated at $37^{\circ} \mathrm{C}$ in a $5 \% \mathrm{CO}_{2}$ incubator for 5 days. MTS was then added into 5 wells each group and incubated for another 4 hours before cell proliferation assay. The assay was performed according to the manufacturer's brochure and OD values were collected at a wavelength of $490 \mathrm{~nm}$. All assays were done in triplicate.

\section{Flow cytometry analysis}

FCM analysis was performed to detect cell cycle distribution and apoptosis. HL-60 cells transfected with shRPL34-lentivirus or shCtrl-lentivirus were incubated in 6-well plates for 5 days, and the medium was removed when the cells reached an enrichment rate of $85 \%$, after which all cells were suspended, centrifuged and fixed with precooled $70 \%$ ethanol for one hour. After wash, the cells were treated with propidium iodide (PI; $50 \mu \mathrm{g} / \mathrm{ml}$, SigmaAldrich ${ }^{\circledR}$ Co. LLC., USA) in the presence of RNase A (100 $\mu \mathrm{g} / \mathrm{ml}$; Fermentas ${ }^{\circledR}$, Shanghai, China). For the detection of cell cycle distribution, the cell suspension was filtered through a 300-mesh, and the DNA content of the stained nuclei was analyzed with BD FACSCalibur flow cytometer (BD Biosciences, San Diego, USA). Each experiment was performed in triplicates. For apoptosis assay, HL-60 cells were collected and washed with PBS at 5 days after transfection with shRPL34 or shCtrl lentiviral vectors. The cells were stained with Annexin V (eBioscience, San Diego, US) at $4^{\circ} \mathrm{C}$ for half an hour before FCM analysis. Each experiment was conducted in triplicate.

\section{Public gene expression datasets of AML}

We downloaded the GSE12417 and GSE2191 datasets from the Gene Expression Omnibus (GEO) (GEO:https:// www.ncbi.nlm.nih.gov/geo/), which revealed the gene expression profile of 163 patients with AML using affymetrix human genome U133A array and 54 pediatric AML patients using affymetrix human genome U95 version 2 
array, respectively. Univariate Cox regression analysis was performed to explore the prognostic value of RPL34 in AML.

\section{Statistical analysis}

One-way ANOVA and Student's t-test were used for raw data analysis. Statistical analysis was performed using SPSS for Windows version 17.0 (SPSS, Inc., Chicago, IL, USA). The statistical data for each group were presented as the mean $\pm \mathrm{SD}$. A value of $P<0.05$ was considered as statistically significant.

\section{Results}

\section{Expression of RPL34 is elevated in AML and corre- lates with inferior outcomes}

We first investigated the transcription level of RPL34 in BM samples of AML patients by RT-qPCR, and as shown in Figure 1A, RPL34 mRNA level was significantly higher than that in normal BM controls. Next, we explored the association between RPL34 expression level and overall survival time in 163 patients with AML (data

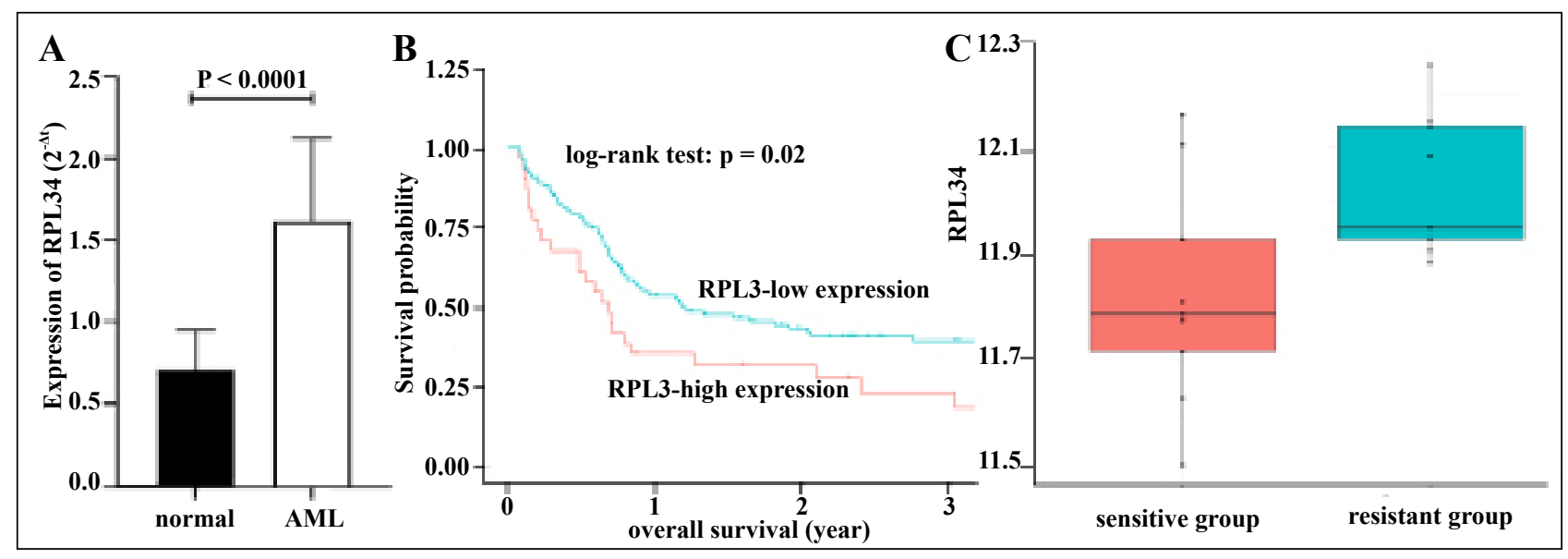

Figure 1. Expression level and prognostic value of RPL34 in AML patients. (A) The mRNA level of RPL34 in BM samples of AML patients was significantly higher than that in normal BM controls. All data represent mean \pm standard deviation. (B) AML patients with high RPL34 expression level had significantly inferior survival outcomes than those with low level (Data was from GSE12417 dataset). (C) AML patients in the resistant group (those who failed induction therapy or relapsed with dismal outcomes) had significantly higher expression level of RPL34 than those in the sensitive group (those who got complete response and had long-term survival) $(P<0.05)$.

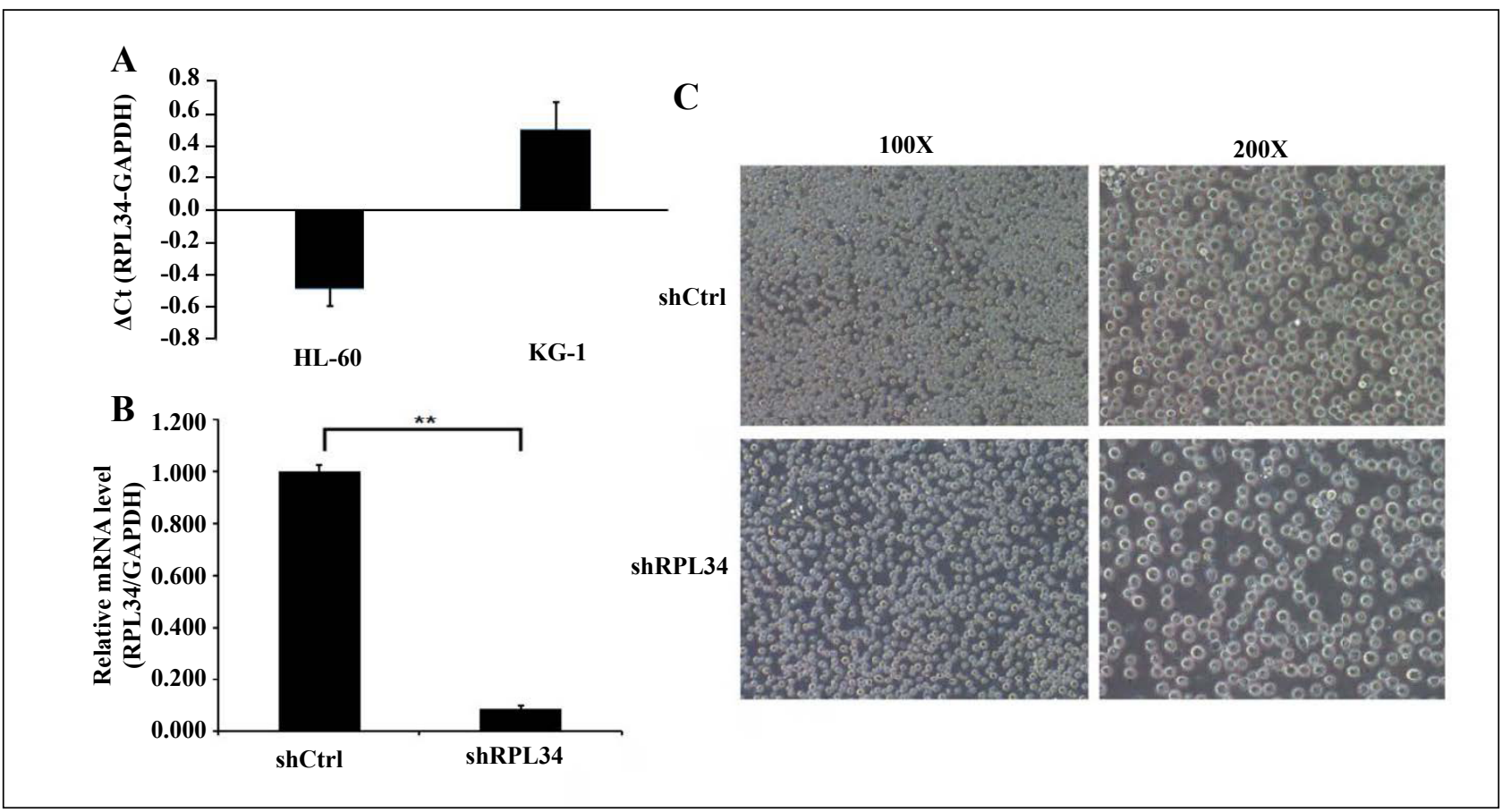

Figure 2. Expression of RPL34 and knockdown efficiency test in AML cell lines. (A) Both HL-60 and KG-1 cell lines had increased expression of RPL34 mRNA detected by RT-qPCR. (B) Transfection with lenti-shRPL34 could effectively decrease the mRNA level of RPL34 by $91.5 \%$. (C) The infection rate of lentivirus in both groups exceeded $80 \%$ at 3 days post transfection. All data represent mean \pm standard deviation; ** $P<0.005$. 


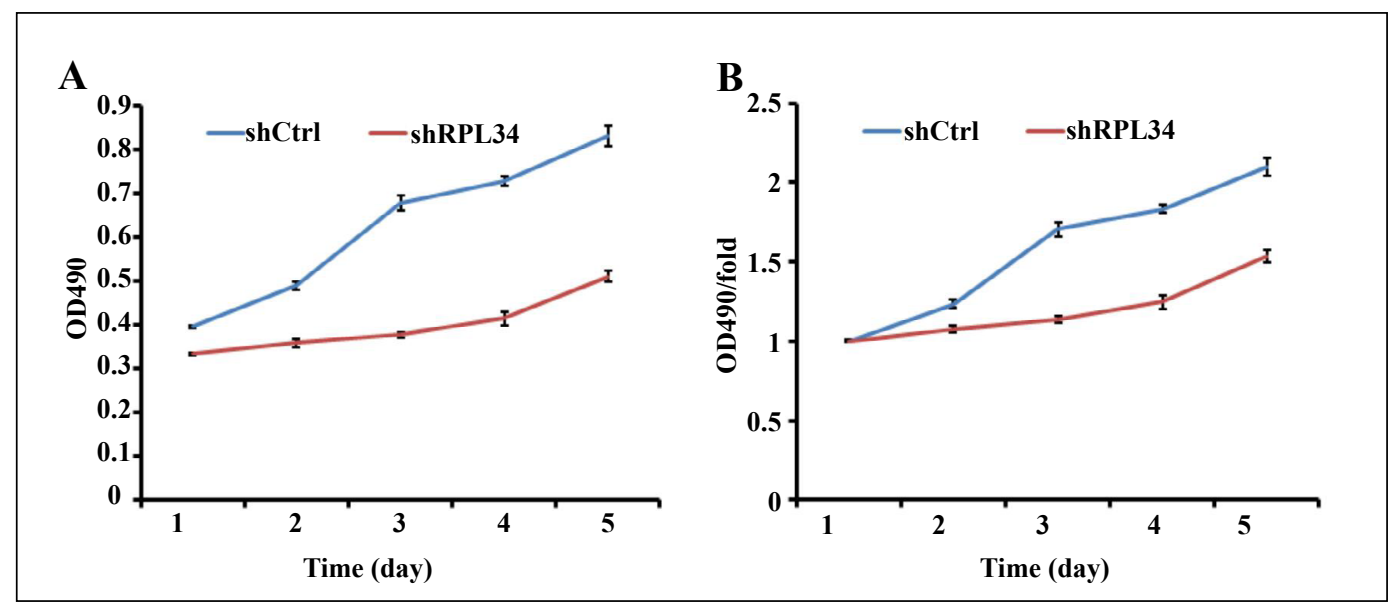

Figure 3. Knockdown of RPL34 inhibited AML cell proliferation in vitro. The effect of RPL34 silencing on HL-60 cell proliferation was measured using MTS assay. ALL experiments were performed in triplicate, and all data represent mean \pm standard deviation; (shCtrl vs. ShRPL34: $P<$ 0.05 ).

from GSE12417 dataset). As demonstrated in Figure 1B, patients with higher expression level of RPL34 (high group) had significantly inferior survival outcomes than those with lower level (low group), which may indicate that RPL34 could mediate the chemoresistance in AML patients and is a valuable prognostic factor. To verify this, we performed analysis using GSE2191 dataset, in which 9 patients were in complete remission and long-term survival (designated as "sensitive" group), and 9 patients had failed induction therapy or relapsed with dismal prognosis (designated as "resistant" group). As is shown in Figure $1 \mathrm{C}$, the expression level of RPL34 in the resistant group was significantly higher than that in the sensitive group $(P$ $<0.05)$, indicating RPL34 may play a critical role in the mechanism of chemoresistance in AML.

\section{Expression of RPL34 in two AML cell lines}

We detected the expression level of RPL34 mRNA in two AML cell lines HL-60 and KG-1 using RT-PCR. As shown in Figure 2A, both cell lines had elevated expression of RPL34 compared with GAPDH.

\section{Determination of knockdown efficiency and lentivirus transfection rate in HL-60 cell line}

HL-60 cell line was transfected with shCtrl lentivirus or shRPL34 lentivirus. As shown in Figure 2B, as compared with shCtrl lentivirus infected cells, the expression level of RPL34 mRNA was reduced by $91.5 \%$ in shRPL34 lentivirus infected HL-60 cells at 5 days after transfection, indicating effective knockdown of RPL34 gene sequence. At 3 days after lentivirus transfection, the proportion of transfected HL-60 cells was more than $80 \%$ for both shCtrl and shRPL34 lentivirus (Figure 2C). Meanwhile, the cell density of shRPL34 lentivirus transfected group seemed to be lower than that of shCtrl group, indicating the potential proliferation inhibition by RPL34 knockdown.

\section{The role of RPL34 in AML cell proliferation}

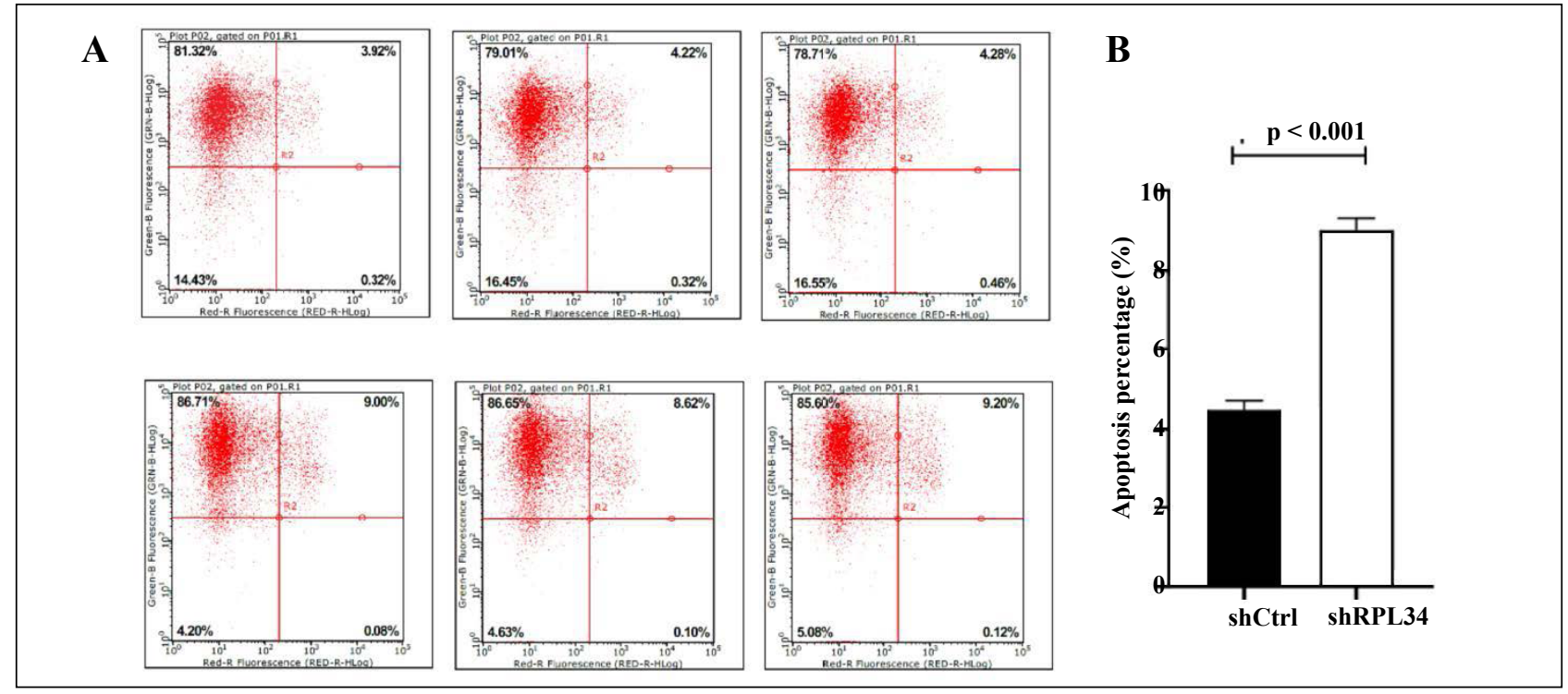

Figure 4. Effect of RPL34 knockdown on apoptosis of HL-60 cells. (A) Apoptotic percentages were determined by Annexin-V staining and FCM. Each group is shown in triplicates. (B) Quantification results demonstrated significant increase in apoptosis in shRPL34 lentivirus transfected HL-60 cells $(P<0.001)$. All data represent mean \pm standard deviation. 


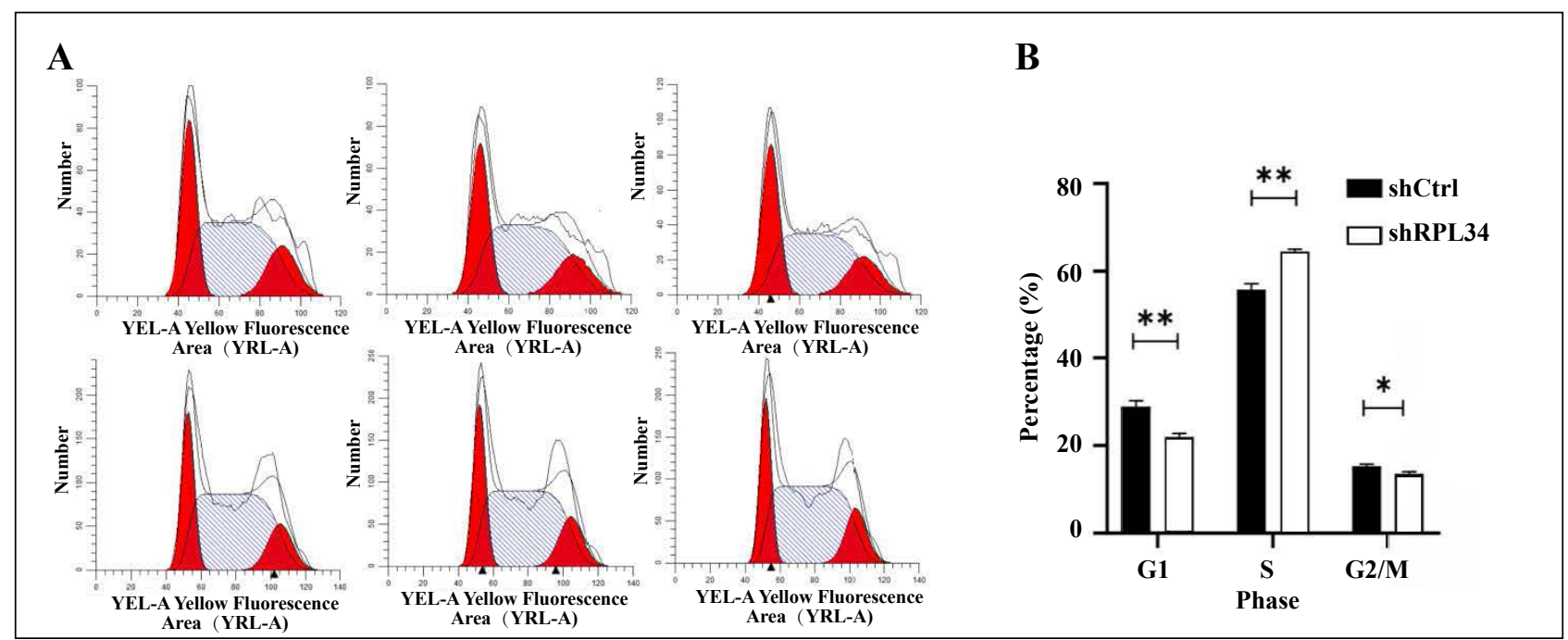

Figure 5. Effect of RPL34 knockdown on cell cycle distribution of HL-60 cells. (A) Cell cycle distribution was analyzed by FCM, and each group is shown in triplicates. (B) Cell cycle was arrested in S phase in the shRPL34 group. All data represent mean \pm standard deviation; $* P<0.05$, ** $P<$ 0.005 .

At 10 days after infection, both HL-60 cells transfected with shCtrl and shRPL34 lentivirus were seeded and incubated for 5 days before cell proliferation analysis. As illustrated in Figure $3 \mathrm{~A}$ and B, the growth rate of shRPL34transfected cells was significantly decreased than the control cells, indicating RPL34 may be involved in the cell proliferation process of AML.

\section{Knockdown of RPL34 promotes AML cell apoptosis}

In order to clarify whether knockdown of RPL34 could promote AML cell apoptosis, we measured the apoptotic percentage of HL-60 cells at 5 days after lentivirus transfection using Annexin V-APC staining and FCM. As is shown in Figure 4, a significant increase in apoptotic percentage was found in shRPL34-transfected HL60 cells $(9.01 \pm 0.30 \%)$ compared to the control group $(4.48 \pm 0.23 \%)(P<0.0001)$, indicating suppression of RPL34 could help triggering the apoptosis of AML cells in vitro.

\section{Knockdown of RPL34 affected cell cycle distribution in AML cells}

Knockdown of RPL34 may inhibit AML cell growth through cell cycle arrest. To test this, we determined the cell cycle distribution mode of HL-60 cells at 5 days after transfection with shCtrl or shRPL34 lentivirus using FCM. As is shown in Figure 5, apparent cell cycle arrest in $\mathrm{S}$ phase was found in HL-60 cells infected with shRPL34 lentivirus. The percentages of different phases in shCtrl and shRPL34 groups were as following: G1 phase, 28.87 $\pm 1.35 \%$ vs. $21.94 \pm 0.83 \%, P=0.0016$; $\mathrm{S}$ phase, $55.84 \pm$ $1.35 \%$ vs. $64.51 \pm 0.55 \%, P=0.0005 ; \mathrm{G} 2 / \mathrm{M}$ phase, 15.29 $\pm 0.45 \%$ vs. $13.55 \pm 0.49 \%, P=0.01$. Thus, RPL34 knockdown could inhibit AML cell proliferation by arresting AML cells in $\mathrm{S}$ phase.

\section{Discussion}

Acute myeloid leukemia (AML) is a highly aggressive disease and results in great mortality among adult patients [1]. Though the generally used $7+3$ regimen can lead to a CR rate of $60-80 \%$, more than half of adult patients eventually die of AML due to chemoresistance [2]. Nowadays, genomic risk stratification is applied to predict the prognosis and guide consolidation strategies for AML patients. However, great heterogeneity still exists among patients within the same stratification. Thus, new biomarkers are needed to better define the prognosis of AML patients, and exploration of novel targets may contribute to overcome the dismal prognosis of AML patients with chemoresistance.

Ribosomal proteins (RPs) play important roles in cell proliferation and growth in most eukaryotic cells [7]. However, in recent years, increasing evidence has shown RPs may involve in the process of tumorigenesis, metastasis, and therapy resistance [7, 9-11]. A few literatures have been reported to reveal the role of RPL34, one type of ribosomal protein, in solid tumors [8-9]. To the best of our knowledge, the present study is the first to investigate the expression and clinical significance of RPL34 in AML. Firstly, by comparing the expression level of RPL34 mRNA in BM of AML and normal controls, we found significant upregulation of RPL34 in AML (Figure 1A). Next, in a large cohort of AML patients, the prognostic role of RPL34 was confirmed, with patients in highgroup having significantly inferior outcomes than their counterparts (Figure 1B). The unsatisfied prognosis may largely result from chemoresistance, and we verified our guess through detailed analysis in another cohort of pediatric AML patients. Indeed, patients who got complete response after induction therapy and enjoyed long-term survival had significantly lower level of RPL34 than those 
that failed induction therapies and suffered from recurrent manuscript.

disease relapses (Figure 1C).

Previous studies have reported that RPL34 promotes tu- References

morigenesis through affecting cell proliferation, apoptosis, and cell cycle distribution [7-9]. In order to define the role of RPL34 in AML, we constructed shRPL34-lentivirus to successfully knockdown RPL34 in HL-60 cell line, a commonly used AML cell line. Consistent with other studies performed in solid tumors [8-9], we found knockdown of RPL34 could significantly inhibit cell proliferation and trigger apoptosis. In cell cycle distribution analysis, more HL-60 cells transfected with shRPL34 lentivirus were arrested in S phase. The normal function of RPL34 is to promote ribosome assembly, and impaired ribosome assembly by silencing RPL34 will generate a feedback signal to cell cycle regulators, which can trigger cell cycle arrest or apoptosis.

In conclusion, the present study demonstrated that downregulation of RPL34 using shRPL34-lentivirus could inhibit cell proliferation, promote cell apoptosis, and induce cell cycle arrest in AML cell line HL-60. Also, its expression and clinical significance in AML patients was confirmed. All these findings suggest that RPL34 may be a potential novel therapy target in AML. Future work should be done to validate our findings and explore its mechanisms of action in mediating chemoresistance.

\section{Declarations}

Acknowledgements: The authors would like to thank the Life-Ontology Biological Technology Co., Ltd for assisting with bioinformatics analysis, and appreciate GeneChem Co.Ltd for experiment guidance.

Author's contribution: LW contributed to the conception and design of the work. JY and HNW carried out the experiments. WDW provided the clinical samples and detailed clinical information. XJL performed bioinformatics analysis. LW and HNW wrote the manuscript. All authors read and approved the final manuscript.

Conflict of Interest: : Liang Wang is a member of the Editorial Board of Aging Pathobiology and Therapeutics. All authors declare no conflict of interest and were not involved in the journal's review or desicions related to this

1. Rebecca L. Siegel, Kimberly D. Miller, Ahmedin Jemal. Cancer statistics, 2017. Ca A Cancer Journal for Clinicians, 2017, 67.

2. Fernandez HF, Sun Z, Yao X, et al. None. Anthracycline Dose Intensification in Acute Myeloid Leukemia. New England Journal of Medicine, 2009, 361(26):2578-2578.

3. Burnett A K, Russell N H , Hills R K, et al. Optimization of Chemotherapy for Younger Patients With Acute Myeloid Leukemia: Results of the Medical Research Council AML15 Trial. Journal of Clinical Oncology, 2013, 31(27):3360-3368.

4. Ravandi F, Alattar ML, Grunwald MR, et al. Phase 2 study of azacytidine plus sorafenib in patients with acute myeloid leukemia and FLT-3 internal tandem duplication mutation. Blood the Journal of the American Society of Hematology, 2013, 121(23):4655-62.

5. Stein E M , Dinardo C D , Pollyea D A, et al. Enasidenib in mutant IDH2 relapsed or refractory acute myeloid leukemia. Blood, 2017, 130(6):722.

6. Das M. Venetoclax with decitabine or azacitidine for AML. Lancet Oncol, 2018, 19(12):e672.

7. Zhou X, Liao WJ, Liao JM, et al. Ribosomal proteins: functions beyond the ribosome. J Mol Cell Biol, 2015, 7(2):92-104

8. Liu H, Liang S, Yang X, et al. RNAi-mediated RPL34 knockdown suppresses the growth of human gastric cancer cells. Oncol Rep, 2015, 34(5):2267-72.

9. Luo S, Zhao J, Fowdur M, et al. Highly expressed ribosomal protein L34 indicates poor prognosis in osteosarcoma and its knockdown suppresses osteosarcoma proliferation probably through translational control. Sci Rep, 2016, 6:37690.

10. Kobayashi T, Sasaki Y, Oshima Y, et al. Activation of the ribosomal protein L13 gene in human gastrointestinal cancer. International Journal of Molecular Medicine, 2006, 18(1):161-170.

11. Mazumder B , Sampath P , Seshadri V, et al. Regulated Release of L13a from the 60S Ribosomal Subunit as A Mechanism of Transcript-Specific Translational Control. Cell, 2003, 115(2):0-198.

Cite this article as: Wang L, Yang J, Wang H, et al. Highly expressed ribosomal protein L34 predicts poor prognosis in acute myeloid leukemia and could be a potential therapy target[J]. Aging Pathobiology and Therapeutics, 2020, 2(1): 32-37. 